\title{
Polymorphism and metastability in NbN: Structural predictions from first principles
}

\author{
Serdar Ögüt and Karin M. Rabe \\ Department of Physics, Yale University \\ P. O. Box 208120, New Haven, Connecticut, 06520-8120
}

(10 May 1995)

\begin{abstract}
We use $a b$ initio pseudopotential total energy calculations with a plane wave basis set to investigate the structural energetics of various phases of polymorphic NbN. Particular attention is given to its recently discovered superconducting phase with a $T_{c}$ of $16.4 \mathrm{~K}$, reported to have the $\mathrm{NbO}$ structure type. Results of total energy calculations show that it is in fact energetically unfavorable for $\mathrm{NbN}$ to form in this cubic structure, and its predicted theoretical lattice constant is significantly smaller than the experimental value. Various approaches to the identification of an alternative structure are discussed. In preliminary investigations, we have found two new structures that are energetically more favorable than the $\mathrm{NbO}$ structure.

61.50.Lt, 74.70.Ad
\end{abstract}

Typeset using REVTEX 
One of the most important advantages of first principles total energy and bandstructure calculations as a tool to understand the physics of materials lies in their applicability to both real and hypothetical systems. For example, with first principles calculations one can identify structures that are energetically competitive with the experimentally realized ones, and such efforts can be quite rewarding if they result in the prediction and identification of new metastable phases. Combined with the recently developed techniques for synthesis and preparation of metastable phases, first principles methods, therefore, prove to be a very useful theoretical tool to investigate the physics of polymorphic materials.

Recently, a new metastable superconducting phase of $\mathrm{NbN}$ with a $T_{c}$ of $16.4 \mathrm{~K}$ has been synthesized using the pulsed laser deposition technique.1 This phase of NbN, which is stabilized by heteroepitaxial growth on a $\mathrm{MgO}(100)$ substrate, forms for a narrow range of substrate temperature, and has been reported to have a primitive cubic lattice based on the presence of (100) and (300) peaks in the diffraction pattern that are forbidden for a facecentered-cubic lattice. Using X-ray diffraction patterns and oscillation photographs from this new $\mathrm{NbN}$ thin film, it has been proposed that it crystallizes in the NbO structure type 2 with a lattice constant of $4.442 \AA$. NbN has long been known to be a highly polymorphic material, with five other crystalline structures already reported in the literature. NbN, AsTi, NiAs, CW, and the well-known superconducting $\mathrm{NaCl}(B 1)$ structure types. Such a high degree of polymorphism, including two superconducting phases with relatively high $T_{c}$ 's, motivates the investigation of the structural energetics of this material and the implications for the occurence of superconductivity. In this paper, we use first principles total energy calculations to understand this interesting system, with particular emphasis on the structure of its recently discovered superconducting phase.

In our calculations, we used the ab initio pseudopotential method with a plane wave basis set and the conjugate gradients algorithm. 4 The general technical issues that arise in the application of this method to metallic systems containing transition metals have been recently discussed in Ref. 5. In this study, for both $\mathrm{N}$ and $\mathrm{Nb}$, we constructed optimized pseudopotentials 6 to perform accurate and efficient calculations with a plane wave basis set. 
For $\mathrm{Nb}$, we used scalar relativistic pseudopotentials, and treated the $4 s$ and $4 p$ semicore states as valence orbitals. These pseudopotentials were put into separable form with two projectors for each angular momentum with $l=1$ and $l=2$ components taken as local for $\mathrm{N}$ and $\mathrm{Nb}$, respectively. In order to obtain a $1 \mathrm{mRy}$ kinetic energy convergence, we performed all calculations at an energy cutoff of $60 \mathrm{Ry}$. All calculations were performed within the local density approximation (LDA) with the exchange-correlation functional of Ceperley and Alder as parametrized by Perdew and Zunger $\mathbf{6}$-point sets were constructed using the Monkhorst-Pack scheme 9 with varying choices of $q$, as will be specified below. To overcome problems associated with finite k-point sampling in metallic systems, we used the thermal broadening scheme of Gillan 10 with broadening energies of $\approx 0.2 \mathrm{eV}$. For cubic structures, the total energies were fitted to the Birch form. For hexagonal and tetragonal lattices, both the unit cell volumes and the $c / a$ ratios were varied, and the energies were fitted to third order polynomials in $c / a$ and to the Birch form in unit cell volume, as described in Ref. 11. After the optimum volumes and $c / a$ ratios were obtained, the $\mathbf{k}$-point grids were increased to check for convergence. Through the use of thermal broadening, the convergence as a function of the number of $\mathbf{k}$ points was found be very good, as expected, with structural energy differences differing only a few meV for larger Monkhorst-Pack grids.

We first performed total energy calculations for the relatively simple phases of $\mathrm{NbN}$ that are observed experimentally, namely the CW, NiAs, and B1 structure types. The CW (space group $P \overline{6} m 2$ ) and NiAs (space group $P 6_{3} / m m c$ ) structures are hexagonal with one and two formula units in the unit cell, respectively. As shown in Table I, the theoretical lattice parameters for these structures were found to be in very good agreement with experiment. Figure 1 shows the results of total energy calculations for these compounds, and it can be seen that the superconducting $B 1$ phase is actually a metastable phase, as already pointed out in Ref. 12. The energy difference we find between the $B 1$ and the $\mathrm{CW}$ phases is 0.31 $\mathrm{eV}$ per formula unit, while the ground-state energies for the CW and NiAs structures are almost the same (differing only by $13 \mathrm{meV}$ ) within the calculational accuracy. Next, we performed total energy calculations for the $\mathrm{NbO}$ structure type (space group $\mathrm{Pm} 3 m$ ), which 
has been reported for the recently discovered superconducting phase of NbN. This structure can be viewed as a vacancy-ordered $B 1$ structure with the atoms at $(0,0,0)$ and $\left(\frac{1}{2}, \frac{1}{2}, \frac{1}{2}\right)$ missing [Fig. 2(a)]. As can be seen in Fig. 3, the calculated energy difference between the $\mathrm{NbO}$ and $B 1$ structure is $0.92 \mathrm{eV}$ per formula unit, indicating that if the new phase had the NbO structure type, it would be extremely high in energy. Moreover, the theoretical lattice constant $4.207 \AA$ is significantly smaller (5.3\%) than the experimental value of 4.442 A. Such a discrepancy between experiment and theory is suspiciously large. First of all, the agreement between our LDA lattice constant for the $B 1$ structure and experiment is very good, with only a $0.5 \%$ underestimate, indicating that LDA errors are quite small. Second, although thin films grown epitaxially tend to have slightly larger lattice constants than their bulk phases, the differences for a number of $\mathrm{NbN}_{x}$ compounds are within 1-2\%. 13 Third, given an experimental lattice constant of $4.39 \AA$ for $\mathrm{NbN}$ in the $B 1$ structure, one would expect that with the removal of $25 \%$ of the atoms, the lattice constant for the $\mathrm{NbO}$ structure would be smaller, not larger, although the volume per formula unit may increase. This expected decrease in the lattice constant is exactly what is observed in our previous calculations for the $\mathrm{NbO}$ compound. Our theoretical values for the hypothetical $B 1$ and actual $\mathrm{NbO}$ structure types of $\mathrm{NbO}$ compound are $4.40 \AA$ and $4.18 \AA$, 14 respectively, while the experimental value for $\mathrm{NbO}$ is $4.21 \AA$. 2 That is, the calculated reduction in the lattice constant of $\mathrm{NbN}$ as the structure is changed from $B 1$ to $\mathrm{NbO}$ is very similar to that of the $\mathrm{NbO}$ compound. Therefore, from both lattice constant values and energetic considerations, our total energy calculations strongly suggest that the crystal structure for the recently discovered superconducting cubic phase of $\mathrm{NbN}$ is not isomorphic to the $\mathrm{NbO}$ structure type.

These considerations have led us to search for other candidate structures for this new phase of NbN. First, we examined all space groups with a primitive cubic lattice in the International Tables, 0 and considered all possible crystal structures with three, four, or five formula units in the primitive cell, since structures with less than three and greater than five formula units would most likely not result in a lattice constant of $\approx 4.4 \AA$. In addition to 
the $\mathrm{NbO}$ and $B 1$ structures, we found two more possibilities, namely the space group P23 with $\mathrm{Nb}$ atoms at the $4(\mathrm{e})$ positions and $\mathrm{N}$ atoms at the 3(c) and 1(a) positions, and the space group $\mathrm{P}_{3} 32$ with $\mathrm{Nb}$ atoms at the $4(\mathrm{a})$ and $\mathrm{N}$ atoms at the $4(\mathrm{~b})$ positions. In the first space group, the $4(\mathrm{e})$ positions $(x, x, x),(x, \bar{x}, \bar{x}),(\bar{x}, x, \bar{x}),(\bar{x}, \bar{x}, x)$ are the vertices of a tetrahedron centered at the origin. For $x=1 / 4$ this results in a zincblende $(B 3)$ structure, which has an fcc lattice. Away from $x=1 / 4$, although the lattice becomes simple cubic, our calculations for $\mathrm{NbN}$ show that this distortion results in a higher energy structure. For the second space group $\mathrm{P}_{3} 32$, with a lattice constant of $4.4 \AA$, some of the interatomic distances are much too short (for example, one Nb-N bond length would have to be 1.55 $\AA$ ), resulting in an energetically very unfavorable structure. Therefore, there is no plausible primitive cubic structure with full occupations of the Wyckoff positions that has a lattice constant close to the experimentally observed value.

One way to resolve this puzzle is to recognize that the available experimental data do not absolutely rule out the possibility of a slightly distorted cubic structure. If we consider tetragonal structures, additional vacancy-ordered derivatives of the $B 1$ structure are allowed which may be energetically more favorable than $\mathrm{NbO}$ and have lattice constants closer to the original. One such structure has already been discussed in the context of the $\mathrm{NbO}$ compound 16 It is obtained from the $B 1$ structure by removing the chain of atoms in the [001] directions, instead of removing the atoms at $(0,0,0)$ and $\left(\frac{1}{2}, \frac{1}{2}, \frac{1}{2}\right)$, which results in the NbO structure type. This alternative structure [Fig. 2(b)] also has three formula units in the primitive cell, however it possesses tetragonal symmetry (space group $P 4 / \mathrm{mmm}$ ) because of the preferred direction along which the atoms have been removed. Although this structure has the same concentration of vacancies as $\mathrm{NbO}$, the fact that entire $B 1$-type layers are left intact can be expected to reduce the collapse of the lattice constant relative to the $B 1$ structure. We minimized the total energy for this $P 4 / m m m$ structure with respect to the $c / a$ ratio and volume. As shown in Fig. 3, the optimum structure is energetically more favorable than the $\mathrm{NbO}$ structure by an appreciable $0.2 \mathrm{eV}$ per $\mathrm{NbN}$. Also, the calculated $a b$-plane lattice constant of $4.275 \AA$ for this structure is significantly larger than that of the 
NbO. The calculated lattice parameter in the $c$-direction is $4.182 \AA$, so that the unit cell volume is $2.6 \%$ larger than $\mathrm{NbO}$, and the $c / a$ ratio of 0.978 is close to 1 . Therefore, the positions of the $\mathrm{Nb}$ atoms, which are most sensitive to the X-rays, are almost identical to those in the $\mathrm{NbO}$ structure. However, the lattice constants of this structure (particularly in the $c$-direction) still show a considerable discrepancy with the experimental observations, and it is still not competitive in energy with the $B 1$ structure (higher by $0.72 \mathrm{eV}$ per formula unit). Therefore, although it may be possible to synthesize this phase under appropriate conditions, we are led to the conclusion that the observed phase is not a vacancy-ordered derivative of the $B 1$ structure.17

In the search for the correct crystal structure of the new phase of $\mathrm{NbN}$, it seems that two complementary approaches should be followed. In the first approach, one can simply concentrate on structures with potentially lower energies by identifying the general trends observed in transition metal nitrides, one of which is the close-packing of the transition metal atoms with $\mathrm{N}$ atoms filling the interstitials. 8 Our calculations along this line found yet another structure that is energetically more favorable than the NbO structure. In particular, the hypothetical $B 3$ structure of $\mathrm{NbN}$ (where the $\mathrm{N}$ atoms are at the tetrahedral interstitials) was calculated to have a lattice constant of $4.71 \AA$ with a total energy $0.18 \mathrm{eV}$ per NbN less than the NbO structure type. Although the appearance of (100) and (300) peaks in the diffraction pattern rules out this fcc structure as a possible candidate for the new NbN, again it may be possible to synthesize it with a suitable substrate and processing conditions. On the other hand, a theoretical lattice constant of $4.71 \AA$ for the ideal four formula unit cubic cell makes a vacancy derivative of this structure much more promising for the experimentally observed new phase of $\mathrm{NbN}$ from the point of view of the lattice constant.

A complementary approach is to concentrate on the crystallographically allowed structures that are compatible with the existing experimental observations. For example, one could consider all tetragonal space groups with $c / a$ ratios close to 1 when the Nb-N interatomic distance is taken as $2.2 \AA$. Another possibility is to allow for fractional occupations 
of the Wyckoff positions. For example, primitive cubic crystal structures with six formula units and equal fractional occupations of the Wyckoff positions may have lattice parameters near the experimentally observed value. This increases the number of candidate structures considerably. Fractional occupations are also an interesting possibility for the vacancy derivatives of the $B 3$ structure. Starting with an ideal $B 3$ structure, removing the Nb atom at $(0,0,0)$, and assigning a fractional occupancy of 0.75 for the $\mathrm{N}$ atoms at the tetrahedral sites results in a three-formula-unit cubic structure that could have a lattice parameter close to $4.44 \AA$, given that the minimum energy lattice parameter for the ideal $B 3$ is $4.71 \AA$. And lastly, there is the possibility that the observed phase is slightly nonstoichiometric.

In summary, we used the pseudopotential total energy method to examine the structural energetics of various real and hypothetical phases of polymorphic NbN. We showed that the $\mathrm{NbO}$ structure, reported for its recently discovered superconducting phase, is energetically quite unfavorable, with a theoretical lattice constant significantly smaller than the experimental value. Moreover, we argued that no primitive cubic structure with full occupations of the Wyckoff positions is a likely candidate for this new phase. In our general search for alternative structures, we found two $\mathrm{NbN}$ compounds (in $P 4 / \mathrm{mmm}$ and $B 3$ structures) that are energetically more favorable than the NbO structure. Although these also do not seem to be likely candidates for the new phase, it may be possible to synthesize them under suitable conditions. We therefore predict that there are other metastable phases of the already highly polymorphic NbN still waiting to be discovered experimentally. We presented some general approaches that can be followed to generate a number of candidate structures for the new superconducting $\mathrm{NbN}$. At this point, more experimental input is needed to narrow down the theoretical possibilities and to achieve a definitive determination of the structure.

We would like to thank R. E. Treece, S. C. Erwin, E. C. Ethridge, L. L. Boyer and A. R. Kortan for useful discussions and preprints. This work was supported by NSF Grant No. DMR-9057442. In addition, K. M. R acknowledges the support of the Clare Boothe Luce Fund and the Alfred P. Sloan Foundation. 


\section{REFERENCES}

${ }^{1}$ R. E. Treece et al., Appl. Phys. Lett. 65, 2860 (1994); Phys. Rev. B 51, 9356 (1995).

${ }^{2}$ A. L. Bowman, Acta Crystallogr. 21, 843 (1966).

${ }^{3}$ P. Villars and L. D. Calvert, Pearson's Handbook of Crystallographic Data for Intermetallic Phases, (American Society of Metals, Metals Park, OH, 1985).

${ }^{4}$ M. C. Payne et al., Rev. Mod. Phys. 64, 1045 (1992).

${ }^{5}$ S. Öğüt and K. M. Rabe, Phys. Rev. B 50, 2075 (1994).

${ }^{6}$ A. M. Rappe et al., Phys. Rev. B 41, 1227 (1990).

${ }^{7}$ L. Kleinman and D. M. Bylander, Phys. Rev. Lett. 48, 1425 (1982); P. E. Blöchl, Phys. Rev. B, 415414 (1990).

${ }^{8}$ D. M. Ceperley and B. J. Alder, Phys. Rev. Lett. 45, 566 (1980); J. P. Perdew and A. Zunger, Phys. Rev. B 23, 5048 (1981).

${ }^{9}$ H. J. Monkhorst and J. D. Pack, Phys. Rev. B 13, 5188 (1976).

${ }^{10}$ M. J. Gillan, J. Phys. Condens. Matter 1, 689 (1989).

${ }^{11}$ M. J. Mehl et al., Phys. Rev. B 41, 10311 (1991).

${ }^{12}$ E. S. Alekseev and A. F. Tatarchenko, Solid State Commun. 73, 569 (1990).

${ }^{13}$ R. E. Treece et al., Chem. Mater. 6, 2205 (1994).

${ }^{14}$ S. Öğüt and K. M. Rabe, (unpublished).

${ }^{15}$ International Union of Crystallography, International Tables for Crystallography, 2nd edition, edited by Theo Hahn (D. Reidel, Boston, 1987), Vol. A.

16 J. K. Burdett and T. Hughbanks, J. Am. Chem. Soc. 106, 3101 (1984).

17 There is one other tetragonal vacancy-ordered derivative of the $B 1$ structure, obtained by 
removing the atoms at $\left(0,0, \frac{1}{2}\right)$ and $\left(\frac{1}{2}, \frac{1}{2}, 0\right)$. However, in this structure none of the $B 1$-type layers parallel to the primitive lattice vectors are kept intact, and our calculations indicate that the lattice constants are smaller than those of the tetragonal structure discussed in the text.

${ }^{18}$ A. F. Wells, Structural Inorganic Chemistry, 5th edition, (Oxford University Press, New York, 1984), pp. 1319-1323. 


\section{FIGURES}

FIG. 1. Total energy versus volume for the CW, NiAs, and B1 structures of NbN. The energies are with respect to the minimum energy corresponding to the LDA lattice parameters of the CW structure. For the CW and NiAs structures, the plotted energies are for the optimum $c / a$ ratio of each volume.

FIG. 2. The crystal structures for the (a) NbO and (b) alternative $P 4 / m m m$ structures (men-

tioned in the text) of $\mathrm{NbN}$. Notice that the $x=\frac{1}{2}$ plane in the $P 4 / \mathrm{mmm}$ structure is left unchanged relative to the $B 1$ structure.

FIG. 3. Total energy versus volume for the $B 1, \mathrm{NbO}$, and $P 4 / m m m$ structures of NbN. The energies are with respect to the minimum energy corresponding to the LDA lattice parameters of the CW structure. For the tetragonal $\mathrm{P} 4 / \mathrm{mmm}$ structure, the plotted energies are for the optimum $c / a$ ratio of each volume. 


\section{TABLES}

TABLE I. The calculated and experimental (in parentheses, taken from Ref. 3) lattice parameters for various real and hypothetical structures of $\mathrm{NbN}$ and the number of $\mathbf{k}$ points used in the calculations. The numbers in parentheses under the fourth column refer to the total number of $\mathbf{k}$ points sampled in the full Brillouin zone.

\begin{tabular}{lccc}
\hline \hline & \multicolumn{2}{c}{ Lattice constants } & \\
Structure & $\mathrm{a}(\AA)$ & $\mathrm{c}(\AA)$ & Number of k points \\
\hline $\mathrm{CW}$ & 2.928 & 2.848 & $17(125)$ \\
& $(2.940)$ & $(2.790)$ & $10(75)$ \\
$\mathrm{NiAs}$ & 2.953 & 5.491 & \\
& $(2.968)$ & $(5.549)$ & $10(256)$ \\
$B 1$ & 4.370 & & $4(64)$ \\
& $(4.391)$ & & $6(64)$ \\
$\mathrm{NbO}$ & 4.207 & & $10(256)$ \\
\hline B3 & 4.275 & 4.182 & \\
\hline \hline
\end{tabular}

